\title{
Unter dem klinischen Bilde einer erworbenen idiopathischen Hautatrophie verlaufende Hyperkeratosis cutis. \\ Von
}

\author{
Dr. Victor Lion, \\ frither Assistenzarzt der Poliklinik.
}

(Hierzu Taf. X.)

Die Thatsache, dass durch die mikroskopische Untersuchung eine auf Grund der Erfahrung nach dem klinischen Bilde anscheinend mit Sicherheit gestellte Diagnose rectificirt wurde und einer andern Platz machte, welche nicht einmal differentialdiagnostisch in Betracht gezogen worden war, dürfte die Veröffentlichung des folgenden Falles, der im Laufe des Sommers 1897 in Dr. Ledermann's Poliklinik beobachtet w urde, rechtfertigen, um so mehr, als auch das klinische Bild einer nicht gerade häufigen Krankheitsform entsprach.

Der Patient war ein 54jähriger Schankwirth, der, abgesehen von einer im 25. Jahre acquirirten Gonorrhoe und öfters wiederkebrenden rheumatischen Beschwerden in Hüften und Beinen, nie krank gewesen sein will. In seiner Familie sind weder Nerven-, noch Haut-, noch sonstige Krankheiten vorgekommen. Pat. ist verheiratet, seine Fran und seine Kinder sind gesund. Er selbst macht einen ausserordentlicb rüstigen Eindruck. An den inneren Organen ist etwas Krankhaftes nicht nachzuweisen. Im Sommer 1896 fiel dem Pat. zuerst eine von ihm selbst nicht näher zu definirende Veränderung beider Hände auf; in April 1897 wurde er auf eine geringfügige Abschuppung der Handteller und Handrücken aufmerksam, und seitdem bemerkte er die allmälige Entwickelung der vorliegenden Affection. In frülerer Zeit - jedoch nur bis etwa zum Jahre 1894 - war er durch seinen Beruf als Wirth gezwungen, die Hände oft in Wasser zu bringen. In den letzten Monaten hat er sie vielfach mit - indifferenten - Salben behandelt. Veranlassung, ärztlichen Rath zu suchen, gab zeitweilig auftretendes Kribbeln und Gefühlsabstumpfung in beiden Händen, die oft "wie eingeschlafen" waren. 
Bei der ersten Untersuchung wurde folgender Status aufgenommen, welcher auch dem jetzt noch bestehenden Krankheitsbilde entspricht:

Es erscheint die Hant der Dorsalseite der Finger, sowie der Handrücken, etwa bis zum Handgelenk, von einem rötblich-gelblichen glänzenden Farbenton. Weiterhin fällt eine besonders an den Fingerrücken und dem distalen Theil des Handrückens äusserst stark entwickelte Faltenbildung auf, die der Haut hier ein pergamentähnliches Aussehen verleiht, während dieselbe sonst am ganzen Körper - von einer noch zu beschreibenden Ausnahme abgesehen - glatt und weich ist und nirgends auch nur Spuren von - etwa durch Alter bedingter - Runzelung aufweist. Diese Falten sind bis zu einem gewissen Grade bleibend, so dass sie sich nur ganz langsam und allmälig ausgleichen, zum Theil werden sie überhaupt nur durch forcirtes Beugen der Hand und der Finger zum Schwinden gebracht. Aber auch nach starker Flexion ist die Haut zu weit für die darunterliegenden Gebilde. An vielen Stellen erscheint sie stark verdünnt, so dass die darunterliegenden Sehnen sichtbar werden und die Hautvenen deutlich durchschimmern. Das Fettgewebe scheint fast völlig zu fehlen. Ueber den Köpfchen der Metacarpophalangealgelenke ist die Haut reichlich mit kleinsten fest adhärirenden Schüppchen bedeckt. Desgleichen sind die sehr derben Handteller leicht schuppend und zeigen mannigfach Schwielenbildung. An den Nägeln ist eine Veränderung nicht wahrzunehmen, ebenso bietet das Haarwachsthum zeine Besonderheiten. Die Schweisssecretion an den Händen ist gering; die Haut ist trocken und fühlt sich kalt an. Das Tastgefühl ist herabgesetzt, so dass das feinere Unterscheidungsvermögen fehlt; sonst ist der neurologische Befund, wie er in der Poliklinik des Herrn Prof. Mendel genau festgestellt wurde, völlig normal.

Die Affection ist an beiden Händen ganz symmetrisch. Eine scharle Begrenzung nach der gesunden Haut findet nicht statt; es geht vielmehr üher dem Handgelenk die glänzende, dünn scheinende Haut allmählig in die weisse Farbe der normalen Haut über. Im übrigen ist die Haut der Arme sowie des Stammes normal. Dagegen befindet sich symmetrisch an beiden Knieca in der Haut unterhalb der Patella je eine circa 5 Markstück grosse, infiltrirte, schuppende Plaque.

Nach diesem Befunde, der, wie auch aus der photographischen Aufnahme ersichtlich ist, kein gewöhnliches Blld ergab, wurde die Diagnose auf erworbene idiopatbische Hautatrophie gestellt, von der bislang nur einige wenige Fälle (Buchwald, ${ }^{1}$ ) Touton, ${ }^{2}$ ) Pospelow, ${ }^{3}$ ) Block, ${ }^{4}$ ) Bronson ${ }^{5}$ )

1) Vierteljahrsschrift f. Dermatologie 1883, pag. 553.

2) Deutsche med. Wochenschrift. 1886, pag. 6 .

3) Annales de Dermatologie et de la Syphiligraphie. 1886, pag. 505.

4) Diss, med. Breslau 1887.

5) Journ. of cutaneous and genito-urinary diseases. 1895, vol. XIII, pag. 1 . 
beschrieben sind. Der Vergleich der klinischen Erscheinungsform des Falles mit diesen bekannten schien die Diagnose zu befestigen. Insbesondere stimmte das Bild unseres Falles mit dem von Touton, bei dem allerdings auch die unteren Extremitäten stärker befallen waren, fast genau überein. Hier wie dort ein sonst gesunder, erblich in keiner Weise belasteter Mann. Die Affection nur an den Extremitäten und an beiden Körperhälften genau symmetrisch. Es hiesse den oben beschriebenen klinischen Befund - die zahlreichen Falten des Handrückens, das Stehenbleiben derselben, das Durchschimmern der Sehnen und Venen, das Verhalten der Sensibilität - fast wiederbolen, sollte Touton's Fall skizzirt werden. Auch erklärte unser Pat. selbst, es komme ihm vor, als sei die Haut der Hände "viel zu weit" geworden, wie Touton den Eindruck hatte, "es stecke die Hand in einem riel zu weiten, schlotterigen Handschuh". Ebenso treffend schien auch bei unserem Falle der Vergleich, den Pospelow angibt, die faltenreiche Haut sehe aus, "wie zwischen den Fingem zerknittertes Cigarettenpapier". Auch sonst zeigt Pospelow's Fall, ebenso wie die anderen, eine weitgehende Aehnlichkeit mit dem unsrigen.

Da wir mithin die Erkrankung unseres Patienten jenen seltenen Fällen zurechnen mussten, so schien es ron Interesse, zumal nur $\mathrm{Buchwald}$ und $\mathrm{Pospel}$ ow eine mikroskopische Untersuchung der erkrankten Haut vorgenommen hatten, eine solche anzustellen.

Es wurde ein kleines längsovales Stückchen - eine jener feststehenden Falten - von der Dorsalseite der linken Hand excidirt. Die Wunde wurde genäht und heilte unter Traumatol per primam. Das herausgeschnittene Hautstückchen wurde theils in Alkohol, theils in $M$ üll e r'scher Flüssigkeit conservirt.

Nach den beiden vorliegenden Untersuchungen sowohl, wie zur Erklärung der verschiedenen klinischen Erscheinungen wurde in der Hauptsache folgender mikroskopischer Befund erwartet: Schwund des subcutanen Fettgewebes, wodurch die Schlaffheit und Weite der Haut, sowie die Runzelung und Faltenbildung entstanden sein mochten; Atrophie der Cutis bei dem Durchscheinen der Gefässe und Sehnen; Reduction des elastischen Gewebes, durch welche das lange Stabilbleiben 
und nur allmälige Zurückgehen der Falten sich erklärte. Des Weiteren wurde bei den erwähnten Untersuchungen Spärlichkeit der Schweissdrüsen, die bei der Kälte der Hände auch in unserem Fall erwartet werden konnte, und der Haarbälge (Buchwald), sowie der Talgdrïsen (Pospelow) beobachtet.

Die Präparate wurden mit Hämatoxylin und mit Alauncarmin, sowie nach Taenzer-Unna mit Orcëin, nach Herxh e im er mit Hämatoxylinlithionlösung gefärbt. Die Untersuchung der Schnitte ergab:

Keine Veränderung der Cutis, keinerlei entzündlichen Erscheinungen; Schweiss- und Talgdrüsen, sowie Haarbälge in normaler Weise vorhanden. Die Epidermis ist auffallend verdickt, das Stratum corneum auf das dreifache seiner gewöhnlichen Stärke vermehrt. Elastische Fasern sind deutlich und in normaler Menge sichtbar, eine Veräıderung ihrer Form und Färbbarkeit ist nicht zu constatiren.

Selbstrerständlich musste nach diesem histologischen Befunde die vorher gestellte Diagnose einer erworbenen idiopathischen Hautatrophie fallen, und diese symmetrische Erkrankung der Hände in die Reihe der Epidermidosen und zwar der reinen Hyperkeratosen eingereiht werden.

Bei Durchsicht der Literatur über diese Krankheitsformen ist es uns nicht gelungen, einen dem hier beschriebenen Fall ähnlichen aufzufinden.

Sucht man nun den histologischen Befund mit dem klinischen Bilde in Uebereinstimmung zu bringen, so lassen sich einige wenig hervorstechende Symptome, wie das glänzende, etwas ins Gelbe spielende Aussehen der Haut, das Kältegefübl in der Hand, sowie auch die leichte Abstumpfung des Gefühls, aus der abnormen Dicke der Hornschicht und aus der vermehrten Proliferation der Epidermiszellen erklären. Schwieriger ist es, die anscheinend so bestimmt für die Atrophie sprechenden Zeichen - die ausserordentliche Faltenbildung, das Stehenbleiben derselben, so dass sie sich nur ganz allmälig wieder ausgleichen, sowie das Durchscheinen der Venen und Sehnen durch die Hautdecke - mit dem Befund der Hyperkeratose in Einklang. zu bringen. 
Die auffallende Faltenbildung ist wohl am einfachsten als auf rein mechanische Weise hervorgerufen zu betrachten. Die starke Proliferation von Epidermiszellen führt zu einer Zunahme der Epidermis sowohl in ihrer Dicke als auch später in ihrer Flächenausdehnung. Dieser Vergrösserung der Fläche vermag die normal gebliebene Cutis nicht zu folgen. Die über das Mass der Cutis hinaus ausgedehnte Epidermis wird dadurch gezwungen, sich in Falten zu legen. Diese Falten besitzen nun einerseits an und für sich in Folge ihrer starken Hornzellendecke ein nur geringes Elasticitätsvermögen, andererseits erscheint das elastische Netz der normalen Cutis nicht mächtig genug, um die übernormale Epidermismasse zu beeinflussen. Aus diesen Gründen müssen die Faltenbildungen bis zu einem gewissen -- hohen -- Grade stabil werden und täuschen so das Fehlen des elastischen Gewebes vor.

Was das Durchscheinen der Venen und Sehnen durch die Haut anbelangt, so muss man annehmen, dass a priori eine relative Magerkeit des Handrïckens vorhanden war, welche von sich aus gestattete, die Venen und Sehnen als blaue, bezw. weisse Stränge durchschimmern zu sehen. In solchem Falle wird, da die Epidermis, wie Kromayer ${ }^{1}$ ) dargethan hat, durchsichtig ist, auch die Auflagerung einer dicken Epidermisschicht an dem optischen Effect nichts ändern. So konnte sich auch dies Symptom als ein Zeichen der Atrophie darstellen und uns keinen Anhalt bieten, schon klinisch die erst mikroskopisch gefundene richtige Diagnose zu stellen.

Bemühen wir uns nun, das so gewonnene Bild unter die bekannten Krankheiten der Haut einzureihen, so muss constatirt werden, dass eine solche Einordnung nicht ohne Weiteres möglich scheint. Immerhin muss bezüglich der Differentialdiagnose zunächst an Ichthyosis circumscripta und an das chronische Eczem gedacht werden. Gegen die erstgenannte Krankheit, mit welcher die vorliegende Affection wohl noch die grösste Aehnlichkeit haben dïrfte, spricht, abgesehen von der Localisation, das Auftreten in spätem Mannesalter und vor allem die ausserordentlich spärliche Desquamation. Auch

1) Deutsche medic. Wochenschrift, 1890, Nr. 25. 
das chronische Eczem ist auszuschliessen, hauptsächlich schon deshalb, weil jeglicher entzündliche Charakter unserer Affection abgeht.

Es wäre dann noch der Sclerodermie und der Xerodermie Erwähnung zu thun. Bei der Sclerodermie, wobei es sich nur um das sogenannte zweite Stadium handeln könnte, wird aber - ausser zahlreichen anderen differentialdiagnostischen Merkmalen - die Haut allmälig so gespannt und so hart, dass es zur Unmöglichkeit wird, eine Falte zu erheben, dass es zur Fixation der Finger in abnormen Stellungen (Sclerodactylie) kommt, während in unserem Falle gerade die excessive Falten. bildung bemerkenswerth war. Ebenso ist bei der Xerodermie, die in den von Kaposi beschriebenen Typen stets in früher Jugend aufzutreten pflegt, die Haut nur schwer faltbar; histologisch zeigt sich dabei die Haut verdünnt. Dieses Merkmal unterscheidet den Fall auch von der Xerodermia acquisita (Auspitz), mit welcher das klinische Bild gleichfalls gewisse Aehnlichkeit zeigt. Eine solche besteht schliesslich, wenn auch in

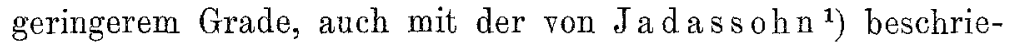
benen Pityriasis atrophicans alba, die jedoch mit Jucken einhergeht und gleichfalls zu Verdünnung und Spannung der Haut führt.

Wir vermögen also die vorliegende Affection, deren Aetiologie nicht völlig klargestellt ist und bei der in Folge dessen die Therapie sich darauf beschränken musste, durch indifferente Salbenbehandlung die Haut möglichst geschmeidig zu machen, nicht einer bekannten Krankheitsform unterzuordnen. Es muss deshalb, da wir uns wohl bewusst sind, mit der Bezeichnung Hyperkeratosis cutis nur einen anatomischen Befund, keine klinisehe Diagnose zu geben, diese in suspenso bleiben, bis weitere Beobachtungen ähnlicher pseudo-atrophischer, in das Gebiet der Hyperkeratosen gehöriger Hauterkrankungen uns näheren Aufschluss geben.

1) Verhandl. d. deutschen dermatol. Gesellschaft, 1891, pag. 392. 


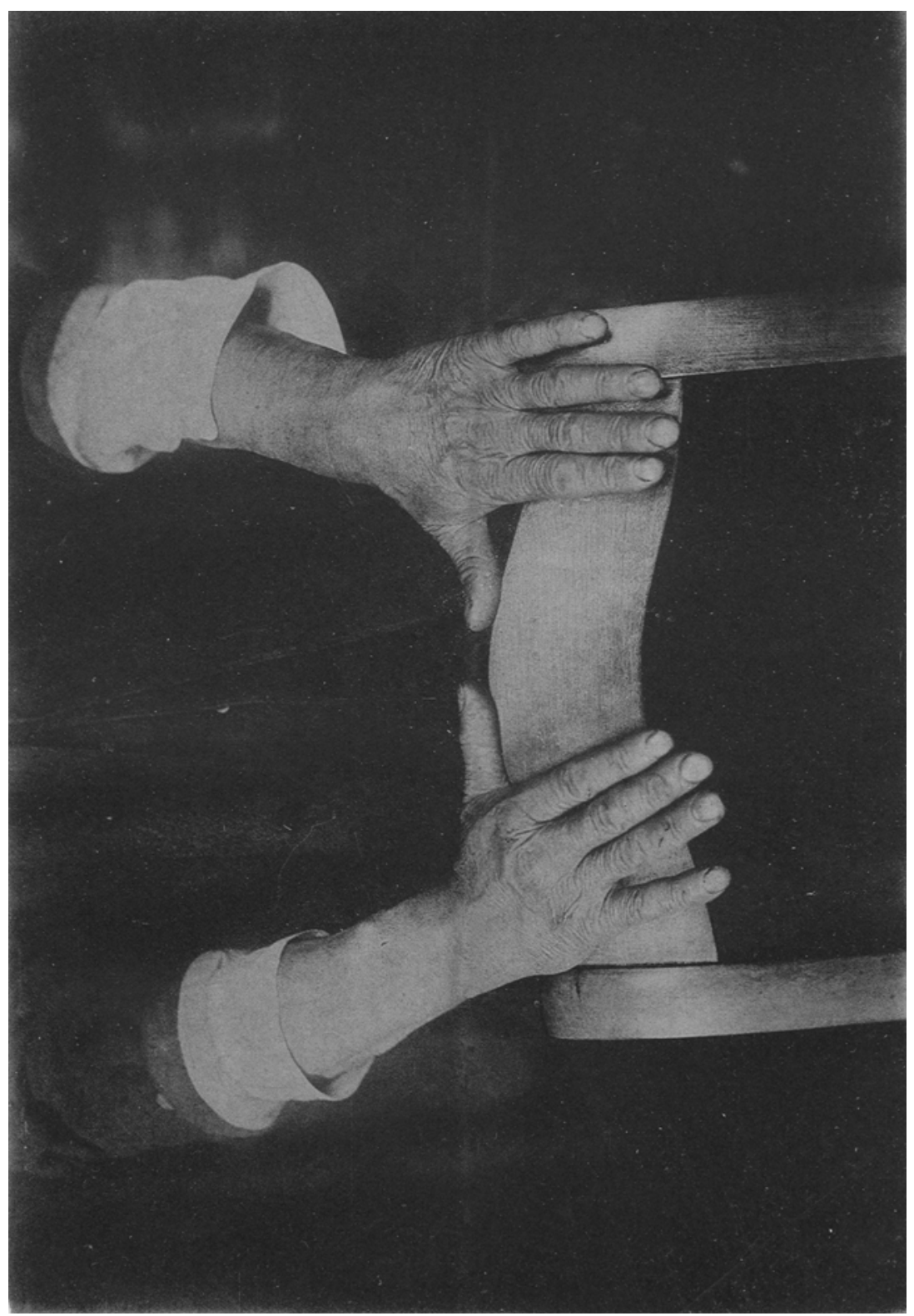

Lion : Typerkeratosis culis.

Ku.k. Hothth A.Hease Prag. 